\title{
Role of Glycated Albumine as a Predictor of Nephropathy in Type 2 Diabetic Iraqi Patients
}

\author{
Ahmed A. kahdim ${ }^{\text {a }}$, Ali Abdulmajid Dyab Allawi ${ }^{\text {b }}$, Shaymaa Z. Nada ${ }^{\text {a }}$ \\ and Kismat M. Turkic, \\ ${ }^{a}$ Department of Biochemistry, College of Medicine ,University of Karbala ,holy Karbala, \\ ${ }^{b}$ FRCP London,Assistant professor and consultant in nephrology and kidney transplantation, Department of internal \\ medicine Baghdad, College of Medicine, University of Baghdad,. ${ }^{c}$ Department of Biochemistry, College of Medicine \\ ,University of Baghdad.
}

\begin{abstract}
:
Background: Diabetic nephropathy is a common complication of diabetes mellitus type2. Glycated albumin level increase in states of abnormally high glucose concentration in diabetes mellitus. The increase in concentration glycated albumin plays role in diabetic nephropathy through stimulation of collagen and fibronectin expression in mesangial cell.

Objective: This study aimed to evaluate the roles of the serum glycated albumin as predictor of diabetic nephropathy with type 2 diabetic patients with normal albuminuria, micro albuminuria and comparing them with normal healthy subjects .

Method: This study was conducted in Medical City, Baghdad Teaching Hospital during the period from December 2015 to June 2016.The study included (90) subjects with age range between (30 - 56) years, divided in to three groups, 30 healthy control, 30 type 2 diabetic mellitus patients with normal albuminuria (micro albumin $<30 \mu \mathrm{g} / \mathrm{ml}$ ) and 30 type 2 diabetic mellitus patients with micro albuminuria (micro albumin $>30 \mu \mathrm{g} / \mathrm{ml}$ ). Body mass index (BMI) was determined, Fasting blood sugar (FBS), serum creatinine, blood urea, and glycated hemoglobin (HbAlc\%) were measured for each individual in addition to the concentration of albumin in urine and estimation glomerular filtration rate (eGFR) was calculated.

Results : The results of the present study showed that the levels of fasting blood sugare, HbAlc \%,blood urea, and serum creatinin, were significantly higher in micro albuminuria than normal albuminuria and healthy control and Glycated Albumin(GA\%) levels showed a statically significant difference ( $p<0.05)$ among the studied groups, it was significantly higher in group micro albuminuria than group normal albuminuria when compared with healthy

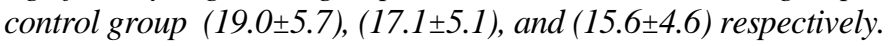

Conclusion: Diabetic patient with micro albuminuria show increase the level of serum GA \% which may be considered as predictor of diabetic nephropathy .
\end{abstract}

Keyword: diabetic nephropathy, micro albuminuria, serum albumin, Glycated Albumin (GA\%)

\section{Introduction}

Diabetic nephropathy is a common complication of diabetes mellitus type2.It is mechanism involved is include changes in blood vessel that supply peripheral nerves (Zychowska et al,2013).Diabetic nephropathy characterized by an increase excretion of protein ,particularly albumin ,decline of the glomerular filtration rate (GFR) and elevated blood pressure that leading to end stage renal failure (Mise et al.,2014).The protein is high sensitive to glycation and used as biomarker of hyperglycemia .The glycation is a non-enzymatic process that sugars molecule and glucose react spontaneously with free amino terminal residues of serum albumins with lysine and arginine ,the condensation of the free aldehyde group of the carbohydrate in it open cyclic form with the N-terminal amino acid of the protein forms (Danese et al.,2015) as we show on figure (1). The measurement of glycated albumin provides information on glucose control mostly limited to the previous 2 weeks but the measurement $\mathrm{HbA1c}$ that provides information on glucose control over the prior 2-3 months (Petrica et al.,2015).The increase in concentration glycated albumin plays role in diabetic nephropathy through stimulation of collagen and fibronectin expression in mesangial cell, other studies that show glycated albumin stimulate reactive oxygen species (ROS) form in mesangial cells (Li and Wang.2010).

\section{Patient and methods:}

This study was conducted in Al kindy Teaching Hospital during the period from December 2015to June 2016.The study included (90) subjects (45 mal and 45female) age is between (30 - 56) years, divided in to three groups of group (1) 30 healthy control, group (2) 30 type 2 diabetes mellitus with normal albuminuria (micro albumin $<30 \mu \mathrm{g} / \mathrm{ml}$ ) and group(3)30 type2 diabetes mellitus with micro albuminuria (micro albumin $>30 \mu \mathrm{g} / \mathrm{ml}$ ) Urine sampling was performed by giving each subject a suitable disposable container with immediate freezing in the deep 
freeze at $-20^{\circ} \mathrm{C}$. The general physical, chemical and microscopic examination of urine were performed on another part of urine specimen to check for the final selection or exclusion of a subject in the study and blood sampling was performed at $8.00-11.00$ am in the fasting state for all the patients and controls and about seven milliliters of venous blood was obtained by antecubital venipuncture using disposable syringes with $\mathrm{G} 21$ needles in the sitting position blood was divided into EDTA tube for hemoglobinA1c test $(2.0 \mathrm{ml})$, Plain tube The blood was allowed to clot for about 20- 30 minutes at room temperature and serum was recovered by centrifugation at $3000 \mathrm{rpm}$ for 10 minutes and transferred in to ependorff tubes and stored at $-20^{\circ} \mathrm{C}$. All patients high and weight were measured body mass index (BMI) was calculate as a ratio of the weight to the high $\left(\mathrm{kg} / \mathrm{m}^{2}\right)$. Blood pressure was measured three time and the average value was considered for data analysis .Fasting blood sugare (FBS) concentration was determined by using glucose oxidase method, HbA1c\% assay using Siemens Dimension clinical chemistry system is an in vitro diagnostic assay for the quantitative determination of HbA1c in human , microalbumin in urine was measured by the fully-auto chemiluminescence immunoassay (CLIA) Analyzer MAGLUMI 1000),Serum urea and serum creatinine levels were determined using an enzymatic colorimetric methods ,eGFR was estimated using the modification of diet in renal disease (MDRD) abbreviated equation : $\left\{\mathrm{GFR}=186 \times(\text { serum creatinin })^{-1.154} \times(\text { age })^{-.203} \times(0.742\right.$ if female $\left.)\right\}$ and serum glycated albumin was measured using Cosbio kit ,enzyme-linked immunosorbent assay (ELISA).

\section{Results}

The ANOVA test showed no statistically significant differences in BMI $(\mathrm{p}>0.05)$ between the studied groups. Systolic and diastolic blood pressure showed statistically significant difference $(\mathrm{p}<0.05)$ among study groups. T- test used showed statistically significant difference in the duration of diabetes among normal albuminuria and micro albuminuria diabetic patient groups. FBS level was significantly higher in micro albuminuria $(\mathrm{p}<0.05)$ than normal albuminuria and healthy control. Glycated hemoglobin ( $\mathrm{HbA1c} \%)$ was significantly higher in micro albuminuria(p $<0.05)$ than normal albuminuria and healthy control group. The levels of blood urea were significantly higher in micro albuminuria ( $\mathrm{p}$-value<0.05) compared with normal albuminuria and healthy control group. Serum creatinine levels was significantly higher in micro albuminuria than normal albuminuria and healthy Group $(p<0.05)$. The mean value of serum glycated albumin was significantly higher in micro albuminuria than normal albuminuria and healthy control group ( $\mathrm{p}<0.05)$ as shown in (Table1) and figure (2)

\section{Discussion}

The presence of micro albuminuria is considered as an indicator or warning signal to renal and cardiovascular disease in patients with type 2 diabetes mellitus, it's also considered as the earliest marker of diabetic nephropathy and associated with significant glomerular damage (Khalaf ,2015)(Ahmed et al,2017). Recent studies showed that the micro albuminuria dose not necessary reflect renal impairment in addition it suggested that the early structure damage in both tubular structure and glomerular may be present in normal albuminuria (Papadopoulou et al.,2015). So there is a need to new find biomarkers that help in identification of the patients risk of the disease and monitoring preventive and therapeutic effects. The result of the present study showed that there is a significant increase in glycated albumin (GA\%) of the diabetic patients with micro albuminuria compared to diabetic patients with normal albuminuria and with healthy group this is in agrees with a study by (Kondaveeti and Shaker., 2013 ). It is noted in (Table 1) there is a slightly increase in GA \% levels in diabetic patients with micro albuminuria and diabetic patients with normal albuminuria . The stander range for glycated albumin by asahi kasei pharma corporation is $11-16 \%$.In this study, GA \% is used as marker of the glycemic control and the occurrence micro and macro vascular complications with diabetic type 2 patients ,HbA1c \% is considered as the gold standard in diabetes control but HbA1c \% level affected by life Spain of erythrocytes and hemoglobin metabolism. HbA1c \% level in the nephropathy dose not any longer correctly show glycemic control. But GA \% is not effected by life spin of erythrocytes and hemoglobin metabolism therefor GA \% is used as the better glycemic marker in kidney failure but factors influence GA\% by an impairment of albumin metabolism such as thyroid dysfunction, nephritic syndrome and liver cirrhosis (Omar et al,2012)(Hasslacher et al.,2014).

\section{Conclusion}

Diabetic patient with micro albuminuria show increase the level of serum glycated albumin which may be considered as predictor of diabetic nephropathy.

No conflict of interest regarding this publication.

\section{Acknowledgement}

No finanicial supports or grants. 


\section{Reference}

[1]. Zychowska M, Rojewska E, Przewlocka B, Mika J. Mechanisms and pharmacology of diabetic neuropathy-experimental and clinical studies. Pharmacological Reports. 2013 Dec 31;65(6):1601-10.

[2]. Mise K, Hoshino J, Ueno T, Sumida K, Hiramatsu R, Hasegawa E, Yamanouchi M, Hayami N, Suwabe T, Sawa N, Fujii T. Clinical implications of linear immunofluorescent staining for immunoglobulin $\mathrm{G}$ in patients with diabetic nephropathy. Diabetes research and clinical practice. 2014 Dec 31;106(3):522-30.

[3]. Danese, E., Montagnana, M., Nouvenne, A. and Lippi, G. Advantages and pitfalls of fructosamine and glycated albumin in the diagnosis and treatment of diabetes. Journal of diabetes science and technology 2015; 9(2), pp.169-176.

[4]. Petrica L, Vlad A, Gluhovschi G, Zamfir A, Popescu C, Gadalean F, Dumitrascu V, Vlad D, Popescu R, Velciov S, Gluhovschi C. Glycated peptides are associated with proximal tubule dysfunction in type 2 diabetes mellitus. International journal of clinical and experimental medicine. 2015;8(2):2516.

[5]. Li Y, Wang S. Glycated albumin activates NADPH oxidase in rat mesangial cells through up-regulation of p47phox. Biochemical and biophysical research communications. 2010 Jun 18;397(1):5-11.

[6]. Khalaf H. Youssef ,2015. Association between Microalbuminuria and glycosylated hemoglobin, some oxidative stress biomarkers and Atherogenicity in type 2 diabetes women .College of medicine and committed of postgraduate studies at AL. Mustansiriya university

[7]. Ahmed A. kahdem, AliA.DyabAllawi, Shaymaa Z. Nada, Kismat M. Turki. Neutrophil gelatinase associated lipocalin (NGAL) in early detection of nephropathy in type 2 diabetic Iraqi patients. Fac Med Baghdad 2017; 59(1),pp.74-78.

[8]. Papadopoulou-Marketou, N., Skevaki, C., Kosteria, I., Peppa, M., Chrousos, G.P., Papassotiriou, I.et al , 2015. NGAL and cystatin C: two possible early markers of diabetic nephropathy in young patients with type 1 diabetes mellitus: one year follow up. Hormones (Athens, Greece), 14(2), pp.232-40.

[9]. Kondaveeti, S.B. and Shaker, I.A., 2013. Evaluation of glycated albumin and microalbuminuria as early risk markers of nephropathy in type 2 diabetes mellitus. Journal of Investigational Biochemistry, 2(2), pp.127-131.

[10]. Omar F.Alazawi,Ali.A.Allawi,Saleh A.Saleh.Prevalance of metabolic syndrome in type 2 diabetic patients in Baghdad teaching hospital.J Fac Med Baghdad. 2012;54(4):281-286.

[11]. Hasslacher, C., Kulozik, F., Platten, I. and Bermejo, J.L., 2014. Glycated albumin and HbA1c as predictors of mortality and vascular complications in type 2 diabetes patients with normal and moderately impaired renal function: 5-year results from a 380 patient cohort. journal of Diabetes Research and Clinical Metabolism, 3(1), p.9.

Table (1) clinical characteristics and baseline investigation of study group

\begin{tabular}{|l|c|c|c|c|}
\hline Characteristics & Healthy group & normal albuminuria & micro albuminuria & P-ANOVA \\
\hline Age (year) & $53.5 \pm 11.7$ & $53.7 \pm 11.5$ & $55.2 \pm 9.7$ & $>0.05$ \\
\hline BMI $\left(\mathrm{Kg} / \mathrm{m}^{2}\right)$ & $27.5 \pm 4.1$ & $29.21 \pm 4.8$ & $29.4 \pm 5.5$ & $>0.05$ \\
\hline BP(systolic)mm/Hg & $124.2 \pm 12.7$ & $133.0 \pm 17.1$ & $92.5 \pm 5.0$ & $<0.05$ \\
\hline BP(diastolic)mm/Hg & $79.5 \pm 8.1$ & $87.0 \pm 5.8$ & $7.9 \pm 5.6$ & \multicolumn{1}{c|}{ t-test $(<0.05)$} \\
\hline Duration DM(year) & - & $5.9 \pm 2.0$ & $236 \pm 83.1$ & $<0.05$ \\
\hline FBS $(\mathrm{mg} / \mathrm{dl})$ & $98.6 \pm 12.4$ & $197.6 \pm 63.0$ & $90.9 \pm 69.0$ & $<0.05$ \\
\hline HbA1c $(\%)$ & $4.8 \pm 0.4$ & $7.5 \pm 0.7$ & $43.2 \pm 17.0$ & $<0.05$ \\
\hline micro albumin $(\mu \mathrm{g} / \mathrm{ml})$ & $5.3 \pm 1.7$ & $18.7 \pm 4.9$ & $1.1 \pm 26.3$ & $<0.05$ \\
\hline Serum urea(mg/dl) & $34.6 \pm 8.3$ & $32.8 \pm 7.1$ & $75.1 \pm 26.3$ & $<0.05$ \\
\hline Serum creatinine $(\mathrm{mg} / \mathrm{dl})$ & $0.7 \pm 18.2$ & $85.6 \pm 21.7$ & $19.0 \pm 5.7$ & $<0.05$ \\
\hline GFR $\left(\left(\mathrm{ml} / \mathrm{min} / 1.73 \mathrm{~m}^{2}\right)\right.$ & $91.6 \pm 18.2$ & $17.1 \pm 5.1$ & & $<0.05$ \\
\hline Serum Glycated Albumin & $15.6 \pm 4.6$ & & & \\
\hline
\end{tabular}

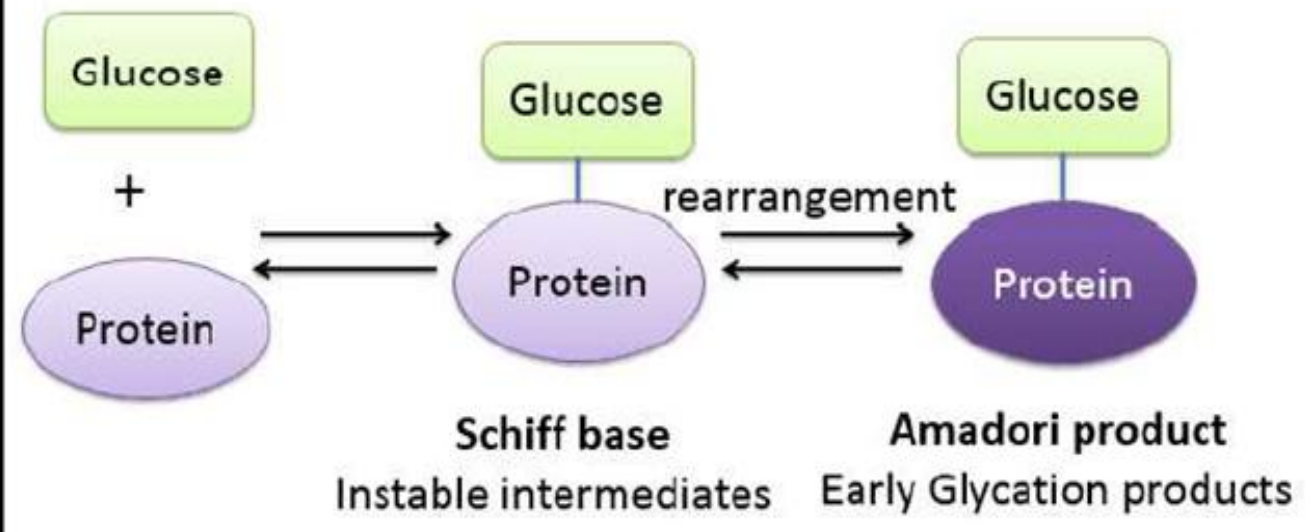

Figure (1) the reaction glucose molecule with protein 


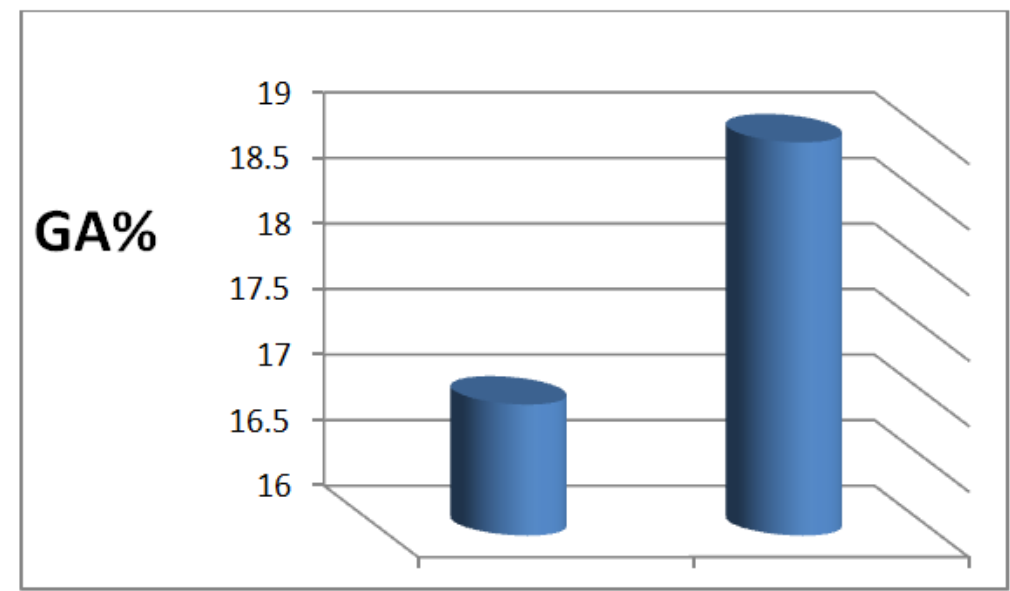

Figure (2) The mean level Glycated Albumin in normal albuminuria and micro albuminuria 